\title{
Facial Expression Recognition: A Survey
}

\author{
Rahul Sharma \\ Student, Department of CSE \\ ABES Engineering College \\ UP, India-201009
}

\author{
Baijnath Kaushik, PhD \\ Associate Professor, \\ ABES Engineering College \\ UP, India-201009
}

\begin{abstract}
Facial expression credit (FEC) is needed to apply the real world. The requests, though, human-computer contact (HCI), psychology and are not manipulated to telecommunications. This is a challenging setback in computer vision and stays alert scutiny case, and a novel method of automatic FEC is counseled to deal alongside the problem. The main trial here, head-currency and non-rigid facial expression (FE) adjustments due to adjustments provoked by the harsh face decoupling to present as they are coupled to the non-linear images. One more trial is how to efficiently order to enable association ponder expression (or disparate facial features) is to exploit the information. FE picture sequence temporal area spatial area data merely emergence, but additionally the progress is not known. Data considering the progress of expression jointly alongside the picture attendance data can more enhance the presentation of recognition. Though, the vibrant data endowed is functional, there how to arrest this data reliably and robustly concerning confronting challenges. For example, a FE sequence normally, one or extra of the main periods of formation and offset top. Provisional data and training in order to arrest and query temporal sequences of comparable data, to make the correspondence amid disparate temporal periods demand to be established. Press can be encoded. In this work, a new vibrant FE, genetic and neural network-based way employing the hybrid procedure is created.
\end{abstract}

\section{Keywords}

FER, FE, FEC, face, HCI

\section{INTRODUCTION}

FE is a vital form of emotional state and mental state. Psychologists notify us that merely $7 \%$ of the finished data such speech, tone, rhythm, etc., but as a proportion of the data bypassed on by the FE of the speech is bypassed, and $38 \%$ is transported by the speech assistant, the $55 \%$ has grasped the whole. Therefore, a lot of priceless data FEC that acknowledges one's consciousness and mental attention is an competent method to become by. Because of this, FEC, vital hypothetical scrutiny worth, useful worth and request worth is displaying existence, has come to be a vital case.

\section{FACIAL EXPRESSION RECOGNITION SYSTEM FRAMEWORK}

Extraction mode, face to FE scrutiny of data, the use of FEC to examine computer thinking and joy, surprise, anger, fear, disgust, sadness and so on people's method of understanding human emotions According to. In finish, the FEC is tear into training and examination, as shown in Figure 1

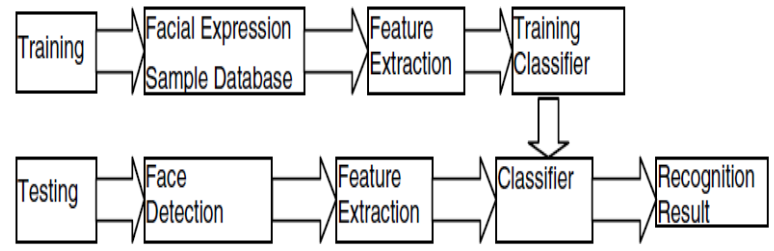

Fig. 1. Facial expression recognition system framework

In the training period, researchers, example pictures to remove features in the early feature association feature classifier to train and demand to become in the end. Assessing periods, beforehand they become the face pictures of the human face is the demand to notice FEs, so that afterward removing features trained classifier, in the end producing aftermath alongside credit of FE association features.

\section{CHALLENGES AND RESEARCH TRENDS}

\subsection{Difficulties in FE Recognition}

There are countless aspects to be learned are: the colossal time-consuming and complex that countless models feature points or specific spans that are marked by hand. Expression pictures, adjustments in the expression of both facial credit and so from time to time encompass data removed features are frequently a blend of adjustments in the expression and individuality attributes. Quite disparate orders and feature extraction (Figure 2) in result at disparate intensities, but a little studies on the face alongside light. Such as the eyes, forehead, unclear as key spans, feature extraction will alter and inhibit alongside the FEC.

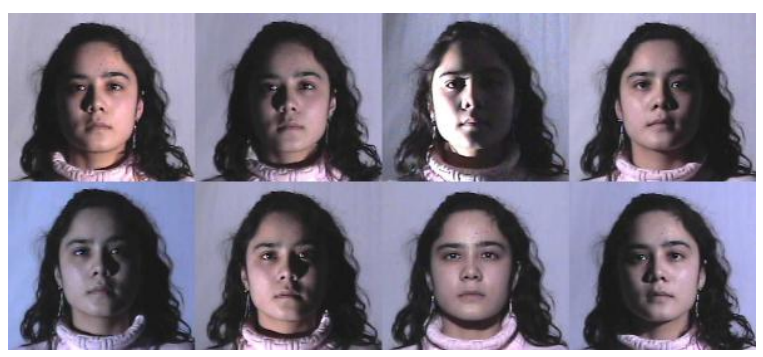

Fig. 2. Subject captured with variant illumination conditions

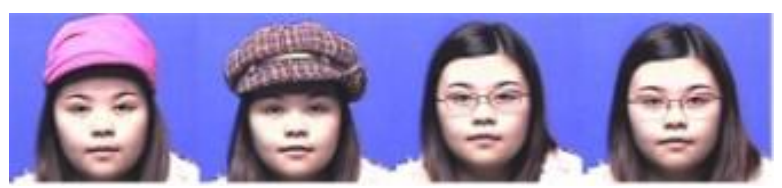

Fig. 3. The subject with different accessories 


\subsection{Development Trend of FE Recognition \\ 1) Measurement of the Distance}

Expression changing pictures encompass data recognizing data and expression. Thus, a competent scope of expression of each two pictures consented and precise meaning needs more discover.

\section{2) Feature Extraction of Micro-expression}

Micro Expression short speech permanent no extra than 1/4S mentions to the automatic identification of the difficulties in this short period and is not facile to be believed, but it can express non-subjective feelings.

\section{3) The Psychological Study}

FE reflects the spirit of the individual, psychological adjustments additionally imitate adjustments in the face. Emotional adjustments, and alongside the vision of how human psychology of features removed employing disparate parameters to examine a convoluted emotional state of mind, but worthwhile trial.

\section{4) Multi-ethnic}

it is valuable to the expression recognition and will become a hotspot to know the relations and different between different ethnic groups' facial features.

\section{5) Three-Dimensional Face Recognition}

3D face recognition, than the two-dimensional light image recognition, face attitude, is vulnerable to the adverse effects of the material covered on the face. 3D database creation FE recognition will provide complete information. It is noteworthy that some 3D database is installed.

\section{6) Face Recognition Based on Multi-feature Fusion}

Multi-feature recognition system is used in order to optimize feature extraction and improve the robustness. How to find a reliable and accurate algorithm for local feature extraction and integration is an important research direction.

7) The Integration of FE Recognition with other Biometric Identification Technologies

Some human biological characteristics are individualities and will not easily change, such as: facial features, eye characteristic (retina, iris), and fingerprint. It should vastly improve identify system performance to mix together face recognition and other biometric identification technologies.

\section{APPLICATIONS}

Automatic FE recognition systems find application in many interesting areas. Recent advances in robotics, especially humanoid robots, with the urgency of the need for a strong expression recognition system is obvious. Speech recognition systems, intelligent visual interface between man and machine will help to create.

Humans communicate effectively and are responsive to each other's emotional states. The computer must also have the ability. Namely, Affective Computing: This is precisely the focus on Human-Computer Interaction research community. Expression recognition plays an important role in recognizing the influence and in turn helps in building meaningful and sensitive HCI interface. The interested reader Zeng et al. refer to the comprehensive survey of the affected-recognition and $\mathrm{HCI}$ on recent advances in their applications to get a complete picture.

The two main applications, namely HCI robotics and sensitive, expression recognition system, telecommunications, behavioral science, video games, animation, psychiatric, automobile safety such as finding other uses in the affected domain host, sensitive music juke box and TV, educational affected software, speech recognition systems, and more realtime and become stronger, we will be seeing many new applications and uses.

\section{CHARACTERISTICS OF A GOOD SYSTEM}

We are now in a position where we can list down the features that a good face expression recognition system must possess:

- It must be fully automatic

- It must have the capability to work with video feeds as well as images

- It must work in real-time

- It must be able to recognize spontaneous expressions

- Along with the prototypic expressions, it must be able to recognize a whole range of other expressions too (probably by recognizing all the facial AUs)

- It must be robust against different lighting conditions

- It must be able to work moderately well even in the presence of occlusions

- It must be unobtrusive

- The images and video feeds do not have to be preprocessed

- It must be person independent

It must work on people from different cultures and different skin colors. It must also be robust against age (in particular, recognize expressions of both infants, adults and the elderly)

- It must be invariant to facial hair, glasses, makeup etc.

- It must be able to work with videos and images of different resolutions

It must be able to recognize expressions from frontal, profile and other intermediate angles head.

\section{RELATED WORK}

Sima, Taheri, Pavan Turaga, et al. (2011) [1] In this paper, FE analysis is one of the important components for effective human-computer interaction. However, to develop robust and generalizable models for expression analysis one needs to break the dependence of the models on the choice of the coordinate frame of the camera i.e. expression models should generalize across facial poses. To perform this systematically, one needs to understand the space of observed images subject to projective transformations. However, since the projective shape-space is cumbersome to work with, this paper addresses the problem by deriving models for expressions on the affine shape-space as an approximation to the projective shape-space by using a Riemannian interpretation of deformations that FEs cause on different parts of the face. Authors used landmark configurations to represent facial deformations and exploit the fact that the affine shape-space can be studied using the Grassmann manifold. This representation enables them to perform various expression analysis and recognition algorithms without the need for the normalization as a preprocessing step. They extend some of the available approaches for expression analysis to the Grassmann manifold and experimentally show promising results, paving the way 
for a more general theory of view-invariant expression analysis.

Yigang, Peng, Arvind Ganesh et al. (2012) [2] This paper studies the problem of simultaneously aligning a batch of linearly correlated images despite gross corruption (such as occlusion). This method seeks an optimal set of image domain transformations such that the matrix of transformed images can be decomposed as the sum of a sparse matrix of errors and a low-rank matrix of recovered aligned images. Authors reduce this extremely challenging optimization problem to a sequence of convex programs that minimize the sum of 11norm and nuclear norm of the two component matrices, which can be efficiently solved by scalable convex optimization techniques. They verified the efficacy of the proposed robust alignment algorithm with extensive experiments on both controlled and uncontrolled real data, demonstrating higher accuracy and efficiency than existing methods over a wide range of realistic misalignments and corruptions.

V Vijayakumari et al. (2013) [3] In this paper, face is the index of mind. It is a complex multidimensional structure and needs a good computing technique for recognition. While using automatic system for face recognition, computers are easily confused by changes in illumination, variation in poses and change in angles of faces. A numerous techniques are being used for security and authentication purposes which includes areas in detective agencies and military purpose. These surveys give the existing methods in automatic face recognition and formulate the way to still increase the performance.

Hassen, Drira, Boulbaba Ben Amor et al. (2013) [4] This paper proposed a novel geometric framework for analyzing 3D faces, with the specific goals of comparing, matching, and averaging their shapes. Paper represents facial surfaces by radial curves emanating from the nose tips and use elastic shape analysis of these curves to develop a Riemannian framework for analyzing shapes of full facial surfaces. This representation, along with the elastic Riemannian metric, seems natural for measuring facial deformations and is robust to challenges such as large facial expressions (especially those with open mouths), large pose variations, missing parts, and partial occlusions due to glasses, hair, and so on. This framework is shown to be promising from both-empirical and theoretical-perspectives. In terms of the empirical evaluation, our results match or improve upon the state-of-the-art methods on three prominent databases: FRGCv2, GavabDB, and Bosphorus, each posing a different type of challenge. From a theoretical perspective, this framework allows for formal statistical inferences, such as the estimation of missing facial parts using PCA on tangent spaces and computing average shapes.

Ashish, Tawari et al. (2013) [5] In this paper, a novel FER framework using audio-visual information analysis is presented. This paper proposed to model the cross-modality data correlation while allowing them to be treated as asynchronous streams. Authors also show that their framework can improve the recognition performance while significantly reducing the computational cost by avoiding redundant or insignificant frame processing by incorporating auditory information. In particular, they design a single good image representation of image sequence by weighted sums of registered face images where the weights are derived using auditory features. They used a still image based technique for the expression recognition task. The framework, however, can be generalized to work with dynamic features as well. They performed experiments using eNTERFACE'05 audio-visual emotional database containing six archetypal emotion classes: Happy, Sad, Surprise, Fear, Anger and Disgust. They present one-to-one binary classification as well as multi-class classification performances evaluated using both subject dependent and independent strategies. Furthermore, they compared multi-class classification accuracies with those of previously published literature which use the same database. Their analyses show promising results.

Steven Lawrence, Fernandes et al. (2013) [6] In real life, images obtained from video cameras or scanners are usually exposed to different levels of noises and blurring effects. This paper proposed a new robust score level fusion technique to recognize faces in the presence of noise and blurring effects. The Proposed Score Level Fusion Technique (PSLFT) is obtained by using combinatory approach and Z-Score normalization using the scores obtained from appearance based techniques: Principal Component Analysis (PCA), Fisher faces (FF), Independent Component Analysis (ICA), Fourier Spectra (FS), Singular Value Decomposition (SVD) and Sparse Representation (SR). The system is trained in the absence of noise, blurring effect but tested by imposing different levels of noises and blurring effects thus they have tried to imitate the real world scenarios. To investigate the performance of PSLFT, authors simulate the real world scenario by adding noises: Median noise, Salt and pepper noise and also adding blurring effects: Motion blur and Gaussian blur. To evaluate performance of the PSLFT, they have considered six standard public face databases: IITK, ATT, JAFEE, CALTECH, GRIMANCE and SHEFFIELD.

S. Elaiwat, Mohammed Bennamoun et al. (2014) [7] In this paper, authors present a robust single modality feature-based algorithm for 3-D face recognition. The proposed algorithm exploits Curvelet transform not only to detect salient points on the face but also to build multi-scale local surface descriptors that can capture highly distinctive rotation/displacement invariant local features around the detected keypoints. This approach is shown to provide robust and accurate recognition under varying illumination conditions and facial expressions. Using the well-known and challenging FRGC v2 dataset, they report a superior performance compared to other algorithms, with a $97.83 \%$ verification rate for probes with all facial expressions.

Seung Ho, Lee, Kostas Plataniotis et al. (2014) [8] In this paper, automatic facial expression recognition (FER) is becoming increasingly important in the area of affective computing systems because of its various emerging applications such as human-machine interface and human emotion analysis. Recently, sparse representation based FER has become popular and has shown an impressive performance. However, sparse representation could often produce less meaningful sparse solution for FER due to intraclass variation such as variation in identity or illumination. This paper proposes a new sparse representation based FER method, aiming to reduce the intra-class variation while emphasizing the facial expression in a query face image. To that end, authors present a new method for generating an intra-class variation image of each expression by using training expression images. The appearance of each intra-class variation image could be close to the appearance of the query face image in identity and illumination. Therefore, the differences between the query face image and its intra-class variation images are used as the expression features for sparse representation. Experimental results show that the proposed FER method has high discriminating capability in terms of 
improving FER performance. Further, the intra-class variation images of non-neutral expressions are complementary with that of neutral expression for improving FER performance.

Yong, Xu, Xiaozhao Fang et al. (2014) [9] In this paper, the image of a face varies with the illumination, pose, and facial expression, thus we say that a single face image is of high uncertainty for representing the face. In this sense, a face image is just an observation and it should not be considered as the absolutely accurate representation of the face. As more face images from the same person provide more observations of the face, more face images may be useful for reducing the uncertainty of the representation of the face and improving the accuracy of face recognition. However, in a real world face recognition system, a subject usually has only a limited number of available face images and thus there is high uncertainty. In this paper, they attempt to improve the face recognition accuracy by reducing the uncertainty. First, they reduce the uncertainty of the face representation by synthesizing the virtual training samples. Then, they select useful training samples that are similar to the test sample from the set of all the original and synthesized virtual training samples. Moreover, they state a theorem that determines the upper bound of the number of useful training samples. Finally, they devise a representation approach based on the selected useful training samples to perform face recognition. Experimental results on five widely used face databases demonstrate that our proposed approach can not only obtain a high face recognition accuracy, but also has a lower computational complexity than the other state-of-the-art approaches.

Bishwas, Mishra, Steven L. Fernandes, et al. (2015) [10] In this paper, facial expression is a way of non-verbal communication. A person depicts his/her feelings through facial expressions. In computer systems facial expressions help in verification, identification and authentication. One popular use of FER is automatic feedback capture from customers upon reacting to a particular product. Effective recognition technology is in high demand by the common users of today's gadgets and technologies. FER technique is broadly classified into two techniques: Feature based techniques and Model based techniques. The key contribution of this article is that authors have analyzed latest state of the art techniques in Feature based techniques and Model based techniques. These techniques are analyzed using various standard public face databases: GEMEP-FERA, BU-3DFE, CK+, Bosphorous, MMI, JAFFE, LFW, FERET, CMU-PIE, Georgia tech, AR, eNTERFACE 05 and FRGC. From the analysis they found that for Feature based Curvelet approach performed on FRGCv2 database gave an excellent $97.83 \%$ recognition rate and Model based textured 3D video technique performed on BU-4DFE database gave an excellent $94.34 \%$ recognition rate.

\section{FACE RECOGNITION}

Several novel ways to solve each change listed above have been proposed. For example, a standard face pattern for template matching methods for computing the correlation of an input image is used for the face detection and localization. Facility irreversible vision eyes, mouth, ears, nose, etc. presence Eigen face neural network based methods and theoretical approaches with the face detection information that is used for detection are used for convenience. However, to fully implement the methods is still a big challenge. Fortunately, the images used in the project have some degree of uniformity, the detection algorithm is simple: First, all the faces are steep and the frontal view; Second, they are almost the same illuminating condition. The project mainly color separation, image segmentation and template matching methods based on face detection technology presents.

\section{FACE RECOGNITION PROCEDURE}

The proposed method involves two steps - encoding and decoding. There are five stages in the encoding phase, the key facial areas including extraction, wavelet transform, the non uniform quantization and choose the level of decomposition, adaptive arithmetic encoding, and field-based encoding hierarchical tree. To help explain the estimate method, wavelet transform; Non - uniform quantization and choose the level of decomposition; Area - based encoding and adaptive arithmetic encoding hierarchical tree faces are set as image compression module. Key areas of the face extraction stage, the projection of the image by using information, the key areas of the face are extracted. And the original image is divided into key areas and backgrounds.

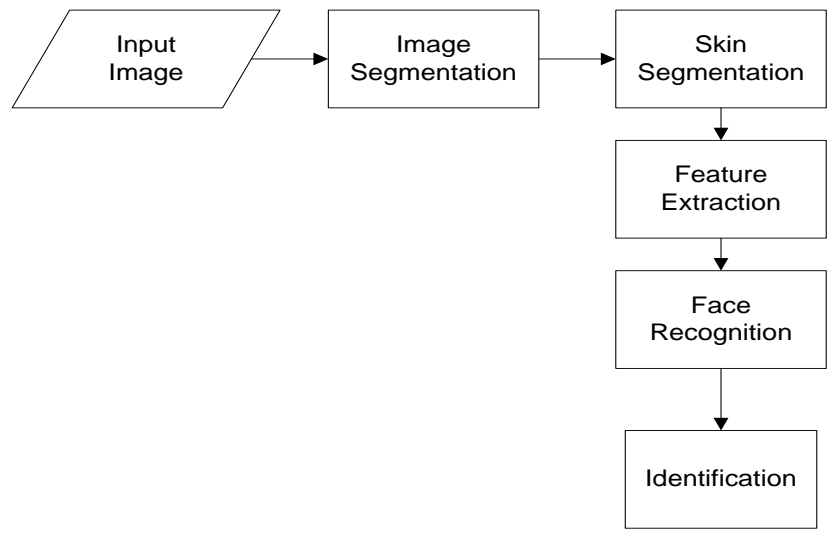

Fig 4: General Architecture of Face Recognition Procedure

Non - uniform quantization step and choose the level of decomposition, the result of the face area of the eye, mouth, nose, and background areas in order arrives. Since the coefficient on the non - core areas is crucial to reduce the human observers, these coefficients using fewer bits without any visual distortion introduced to the human eye can be quantized. Therefore, the coefficients in the various critical areas will be non - equally according to their visual importance quantized.

Key areas and backgrounds, based on the locations of an area - based hierarchical tree coding scheme for adaptively compression bit rate is put to good use on the wavelet coefficients. The higher the bit rate, the key area is the use of low bit-rate background areas, while there. We have diversified beyond the level of decomposition vary significantly between the regions to develop a bit allocation strategy.

The results of the identification of key areas for individual eyes, mouth and nose as ordered. Thus, the level of decomposition of the wavelet coefficients for the eyes to match the compression is kept only part of the nose and mouth of the wavelet coefficients are used for compression. The large image reconstruction to achieve the quality of low bit rate and at the same time to maintain. Hierarchical tree based coding - to further reduce coding redundancy, an adaptive arithmetic coding method is useful on sector output bit stream. Decoding stage, above - mentioned steps different from non - uniform quantization, select the level of decomposition and feature extraction steps to reconstruct facial images are executed in reverse order. 


\section{CONCLUSION AND FUTURE SCOPE}

In recent years, the dynamic $\mathrm{FE}$ recognition have become a new research topic and receives more and more attention. The problem is different from the recognition of static images, dynamic FE recognition of the physical object of an image sequence of a subject captured during the process of FE FE type inference. FE image sequence temporal domain spatial domain information only appearance, but also the development is not known. Information regarding the development of expression together with the image presence information can further enhance the performance of recognition. However, the dynamic information provided is useful, there how to capture this information reliably and robustly about facing challenges.

Eigen face of the techniques used in the experiment system, and then segments the head, eyes, lips and chin detects facial image using. Sobel operator is applied to the image to get the binary histogram. Then use the area as the feature vector, system training and information from the database by taking both live camera is used. The final step was to test images using two approaches; Genetic algorithms and neural network. This was $97.547 \%$ accuracy by genetic algorithms and neural networks that hybrid technology outperforms existing approaches

\section{REFERENCES}

[1] Sima, Taheri, Pavan Turaga, and Rama Chellappa. "Towards view-invariant expression analysis using analytic shape manifolds." In Automatic Face \& Gesture Recognition and Workshops (FG 2011), 2011 IEEE International Conference on, pp. 306-313. IEEE, 2011.

[2] Yigang, Peng, Arvind Ganesh, John Wright, Wenli Xu, and Yi Ma. "RASL: Robust alignment by sparse and low-rank decomposition for linearly correlated images." Pattern Analysis and Machine Intelligence, IEEE Transactions on 34, no. 11, 2233-2246, 2012.

[3] V Vijayakumari. "Face recognition techniques: A survey." World Journal of Computer Application and Technology 1, no. 2, 41-50, 2013.
[4] Hassen, Drira, Boulbaba Ben Amor, Anurag Srivastava, Meroua Daoudi, and Rim Slama. "3D face recognition under expressions, occlusions, and pose variations." Pattern Analysis and Machine Intelligence, IEEE Transactions on 35, no. 9, 2270-2283, 2013.

[5] Ashish, Tawari, and Mohan Manubhai Trivedi. "Face expression recognition by cross modal data association." Multimedia, IEEE Transactions on 15, no. 7, 1543-1552, 2013.

[6] Steven Lawrence, Fernandes, G. Josemin Bala, P. Nagabhushan, and Sajal Kumar Mandal. "Robust face recognition in the presence of noises and blurring effects by fusing appearance based techniques and sparse representation." In Advanced Computing, Networking and Security (ADCONS), 2013 2nd International Conference on, pp. 84-89. IEEE, 2013.

[7] S. Elaiwat, Mohammed Bennamoun, Farid Boussaid, and A. El-Sallam. "3-D face recognition using curvelet local features." Signal Processing Letters, IEEE 21, no. 2, 172$175,2014$.

[8] Seung Ho, Lee, Kostas Plataniotis, N. Konstantinos, and Yong Man Ro. "Intra-class variation reduction using training expression images for sparse representation based facial expression recognition." Affective Computing, IEEE Transactions on 5, no. 3, 340-351, 2014.

[9] Yong, Xu, Xiaozhao Fang, Xuelong Li, Jiang Yang, Jane You, Hong Liu, and Shaohua Teng. "Data uncertainty in face recognition." Cybernetics, IEEE Transactions on 44, no.10, 1950-1961, 2014

[10] Bishwas, Mishra, Steven L. Fernandes, K. Abhishek, Aishwarya Alva, Chaithra Shetty, Chandan V. Ajila, Dhanush Shetty, Harshitha Rao, and Priyanka Shetty. "Facial expression recognition using feature based techniques and model based techniques: A survey." In Electronics and Communication Systems (ICECS), 2015 2nd International Conference on, pp. 589-594. IEEE, 2015. 